\title{
Neutron Imaging: A Non-Destructive Testing Method to Investigate Canned Exhaust After-Treatment System Components for the Three Dimensional Soot, Ash, Urea and Coating Distributions
}

\author{
Christian Gruenzweig, David Mannes, and Florian Schmid
}

Paul Scherrer Institute

\section{Rob Rule}

Ceramex Ltd.

CITATION: Gruenzweig, C., Mannes, D., Schmid, F., and Rule, R., "Neutron Imaging: A Non-Destructive Testing Method to Investigate Canned Exhaust After-Treatment System Components for the Three Dimensional Soot, Ash, Urea and Coating Distributions," SAE Technical Paper 2016-01-0985, 2016, doi:10.4271/2016-01-0985.

Copyright (C) 2016 SAE International

\begin{abstract}
Neutron imaging (NI) is an alternative non-destructive inspection technique compared to the well-known X-ray method. Although neutron imaging data look at a first glance similar to X-ray images it must be underlined that the interaction mechanism of the sample material with neutrons differs fundamentally. X-ray interaction with matter occurs with the electrons in the atomic shells whereas neutrons interact only with the atomic nuclei. Hence, both methods have a different and to great extent complementary contrast origin. Neutron imaging allows for a higher penetration through heavier elements (e.g. metals) whereas a high contrast is given for light elements (e.g. hydrogen).

By the use of neutrons instead of X-rays exhaust after-treatment systems can be successfully examined non-destructively for their soot, ash, urea and coating distributions. The big advantage of neutron imaging is that detailed, high-contrast images can be obtained even in canned substrates (silicon carbide or cordierite). Neutron imaging can be applied to substrates of passenger cars, trucks and even heavy duty vehicles. We can offer a maximum field of view up to $400 \mathrm{~mm} \times 400 \mathrm{~mm}$ and a spatial resolution down to $13,5 \mu \mathrm{m} /$ pixel.

The paper briefly outlines the method of neutron imaging and its advantage and complementary character compared to the conventional X-ray method. We present neutron tomography results of diesel particulate filter (DPF) and gasoline particulate filters (GPF) concerning: (i) Soot, ash, metallic particle and coating distributions in a canned passenger car DPF. (ii) Urea distributions in passenger car DPF. (iii) Ash distribution in a canned passenger car GPF. (iv) Ash residuals of a truck DPF after cleaning with different techniques.
\end{abstract}

\section{Introduction}

Diesel particulate filters (DPF) are commonly used in passenger cars, trucks and heavy duty vehicles to fulfill the restriction on the particulate matter. However it is still difficult to measure the soot distribution within the filter especially when the substrates are canned [1]. Presently, the total amount of soot and ash is only measured by means of simple weighing. X-ray imaging is a conventional nondestructive imaging method mainly used to obtain volumetric information concerning the ash distribution in the filter where the samples are often de-canned to get reasonable information. The soot distribution information however stays unknown. Truck and heavy-duty DPFs are commonly cleaned for the ash residuals. Hereby, the cleaning efficiency is of interest. Hence, to obtain this information, the samples cannot be de-canned, as they will be reinstalled into the vehicles after the cleaning procedure. Hence, $\mathrm{X}$-ray imaging will suffer again from the problem of the canning due to insufficient transmission.

The legislation becomes also more stringent concerning particulate mass emission of gasoline vehicles such as passenger cars, which will possibly be equipped with gasoline particulate filters (GPF). Similar as for the investigation of DPFs, the ash residuals in canned GPFs are challenging to be visualized by X-ray inspections.

The reduction of the nitrogen oxides $\left(\mathrm{NO}_{\mathrm{x}}\right)$ is nowadays a common topic for all diesel engines. Here, the injection of a liquid urea solution is used. The distribution of this solution after the injection often stays unknown, and X-ray inspection will finally fail, as no sensitivity to the water and ammonia solution is obtained. 
In the following we introduce the neutron imaging technique as a non-destructive imaging method, which represents an alternative to well-known X-ray technique. Using neutrons instead of X-ray allows the successful investigation of canned samples for the soot, ash, coating and urea distributions in DPF and GPF applications.

\section{Neutron Imaging and Its Benefit Compared to X-Ray Imaging}

The reason why neutron imaging is much less common as nondestructive testing method in research and for industrial applications can be found in the limited number of sufficiently strong neutron sources and suitable installation dedicated for this challenging technique [2]. Presently, only a dozen facilities worldwide possess of an adequate performance for studies on competitive high level. Nevertheless, NI has proved to be a powerful, competitive and promising method for material research, for many industrial applications and as a tool for different branches in fundamental research.

NI as a technique for non-destructive testing has its application range where the more common X-ray methods will come to their limits: transmission through thick layers of heavy metals, detection of small amounts of hydrogenous materials and some other light elements like Boron or Lithium, or neighboring elements such as e.g. Rhodium and Palladium. Figure 1 shows a comparison of the X-ray $(\mathrm{E}=120 \mathrm{keV})$ and neutron $(\mathrm{E}=25 \mathrm{meV})$ attenuation coefficients $\Sigma$ for different elements of the periodic table.
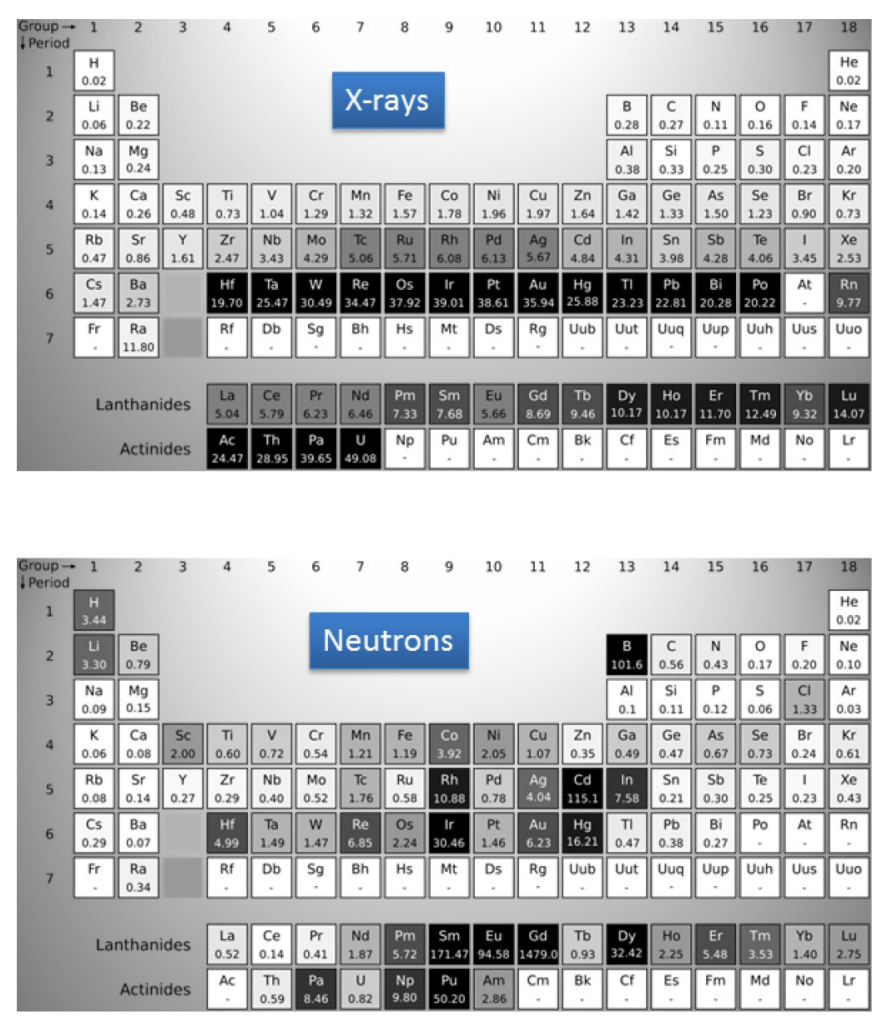

Figure 1. Comparison of the interaction of X-rays (top) and neutrons (bottom) with different elements. The darker an element appears the stronger it attenuates. The values given are the so-called attenuation coefficient.

Although neutron imaging data look at a first glance similar to X-ray images it must be underlined that the interaction mechanism of the sample material with neutrons differs fundamentally from photon interaction. For illustration reasons we compare two tomography data set of a Swiss army knife, as shown in Figure 2 on the top part. X-ray interaction with matter occurs with the electrons in the atomic shells of the inspected materials. Therefore, the interaction probability is directly proportional to the number of electrons, namely the atomic number. Light elements, like hydrogen, have little chance to attenuate $\mathrm{X}$-rays, whereas heavy elements absorb a lot. This has specific consequences for transmission and contrast: hydrogen is nearly invisible, but metallic layers attenuate the X-ray beam to a large extent.

Neutrons interact principally only with the atomic nuclei of the tested materials. Most of the relevant materials scatter neutrons at the nuclei, but there are some prominent materials with huge attenuation capability like Gadolinium, Cadmium, Boron and Lithium. Thermal and cold neutrons have the ability of higher penetration through heavy elements (e.g. metals) whereas a high contrast is given for light materials such as hydrogen or hydrogen containing materials like plastic, as shown in Figure 2 on the bottom.

Hence, it depends on the elemental composition of the studied object or material whether X-ray or neutron imaging will be more the appropriate testing method.
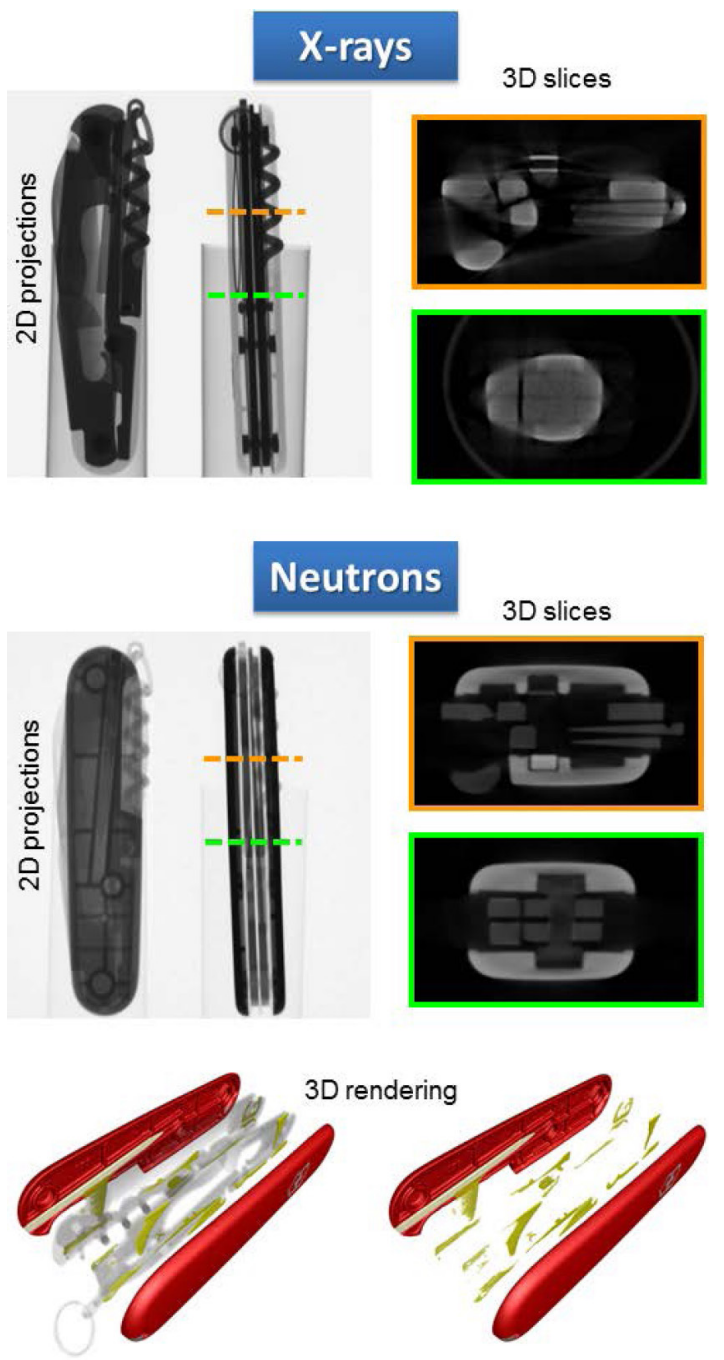

Figure 2. Comparison of the X-ray (top) and neutron (bottom) tomography data of a Swiss army knife. The 3D slices of the X-ray data set are artifacts afflicted caused by the metal components whereas the plastic parts are not visible. In the neutron tomography results, both the metallic parts as well as the plastic parts are visible. Even the thin lubrication film between the metallic parts can be made visible in the $3 \mathrm{D}$ rendering of the neutron data set. 
At the spallation neutron source SINQ at the Paul Scherrer Institut (PSI) mainly three different imaging setups for radiographic and tomographic investigations are available:

- Maxi-setup with a maximum field of view of $400 \times 400 \mathrm{~mm}^{2}$ and a resolution of $150 \mu \mathrm{m} / \mathrm{pixel}$.

- $\quad$ Midi-setup with a field of view ranging from $30 \times 30 \mathrm{~mm} 2$ up to $150 \times 150 \mathrm{~mm} 2$ with a resolution ranging from $30 \mu \mathrm{m} /$ pixel up to $75 \mu \mathrm{m} /$ pixel.

- $\quad$ Micro-setup with a field of view of $27 \times 27 \mathrm{~mm} 2$ and a pixel size of $13.5 \mu \mathrm{m} /$ pixel.

\section{Soot, Ash, Metallic Particle and Coating Distributions in a Canned Passenger Car DPF}

The neutron tomography data of the entire canned DPF are presented in Figure 3. The overview neutron tomography providing a threedimensional volumetric view, with a resolution of $150 \mu \mathrm{m} / \mathrm{px}$, is shown in Figure 3(a). A special feature of this DPF is the additional mounting of an inlet-sided centric masking, which was installed before the last loading phase. This coverage is blocking partially the exhaust flow onto the monolith. The diameter of the mounting was chosen such that the central segment is completely protected and its surrounding segments were only partially loaded. The outer segments were exposed to the full exhaust stream. For the NT measurement 625 projections over an angular range of $360^{\circ}$ were taken with an exposure time of $20 \mathrm{~s}$ per projection.
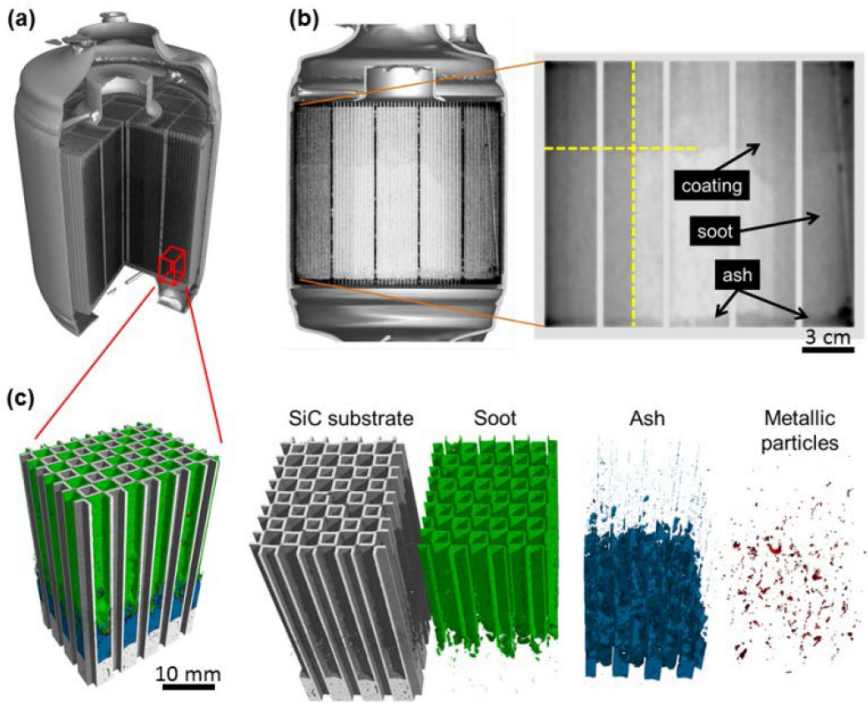

Figure 3. (a) Neutron tomography data of a loaded DPF acquired with the maxi-setup. The canning is no barrier for neutrons and allows an insight into the loaded substrate. (b) Vertical, center slice through the DPF. For a better representation of the soot and ash deposit, the image was processed by a Gaussian filter, which eliminated the channel structures. Clearly visible along the yellow dashed vertical line are the sharp boundaries between loaded and unloaded filters regions. Similarly, a horizontal line is seen in the upper third, which is due to the catalytic zone coating. (c) High-resolution neutron tomography recorded with the micro-setup showing the volume rendering and the segmentation of the individual components. Source: [ $\underline{3}$ ] modified; An animation of the high-resolution tomography can be downloaded from [4].

A more detailed analysis of the local soot and ash deposits as well as the coating distribution is shown in Figure 3(b). Here a vertical section through the middle of the filter is presented and for a clearer representation, the monolith section was processed with a Gaussian filter, which eliminates the channel structures. The darker the areas appear the more abundant is the deposit. Two areas are particularly noticeable: Firstly, a vertically extended region below the coverage running through the whole monolith, which is separated by two clear vertical lines from the rest of the monolith. This area is brighter meaning there is less or no soot and ash deposits. Secondly, a horizontally extended region in the upper third of the filter, which is due to the catalytic zone coating. The trend shows that the outer regions experienced a more abundant loading as expected. Noticeable in Figure $\underline{3(b)}$ is the area below the cover at the bottom of the filter segments. These deposits are ash residues originating from the two regenerations, where the filter was loaded without using of the coverage.

For the high-resolution tomography with the micro-setup (resolution $13.5 \mu \mathrm{m} / \mathrm{px}$ ), the sample had to be cut to fit the size to the field of view of the detector $(27 \mathrm{~mm} \times 27 \mathrm{~mm}) .625$ projections over an angular range of $360^{\circ}$ with an exposure time of $90 \mathrm{~s}$ per projection were recorded. The three-dimensional representation of the tomography data is shown in Figure 3(c). With the help of the tomography data, the different materials could be segmented. This investigation is described in detail in [ $\underline{3}]$. An animation of the high-resolution tomography can be downloaded from [4].

\section{Urea Distribution on DPFs}

Diesel exhaust fluid (DEF) also known as AdBlue is a standardized aqueous urea solution made with $32.5 \%$ urea and $67.5 \%$ demineralized water. DEF is used for the selective catalytic reduction (SCR) process to reduce the NOx concentration in the diesel exhaust emissions. The chemical formula of urea is $\mathrm{CO}\left(\mathrm{NH}_{2}\right)_{2}$, hence contains a considerable amount of hydrogen. As seen in Figure 1, neutrons are very sensitive to hydrogen in contrast to X-rays. As a reference point, $1,3 \mathrm{~cm}$ of water attenuates a thermal neutron beam to $99 \%$. Therefore it is obvious, that the urea solution is a very pronounced contrast agent, and therefore even the diluted spray can be made visibly using neutron imaging. In Figure 4 we present the results of the urea distribution of three pieces of loaded SiC substrates of a DPF. For the NT measurement 625 projections over an angular range of $360^{\circ}$ were taken with an exposure time of $10 \mathrm{~s}$ per projection. The urea solution was applied in three different ways as noted in Figure 4(a): (i) spraying of the urea solution onto the sample and at the same time having a small underpressure applied. (ii) dipping the substrate into $40 \mathrm{~mL}$ of urea solution for 20 minutes. (iii) dipping the substrate into $1 \mathrm{~mL}$ of urea as long as all solution was sucked into the sample. The corresponding $2 \mathrm{D}$ neutron radiography results are shown in Figure 4(b). The darker areas represent the urea solution deposits. In the left and right image the penetration profile of the urea solution from the bottom of the samples is shown, clearly showing the difference between moistened and untainted filter material. The substrate in the middle in Figure 4(b) shows a complete distribution of the urea solution over the whole piece. Figure 4(c) shows the 3D neutron tomography results of the urea distribution (colored in red) of the sample marked in the orange box in Figure 4(b). The left image in Figure 4(c) shows a vertical section through the substrate, the middle one a volume rendered view, and the right on the pure $3 \mathrm{D}$ urea distribution in the sample. 


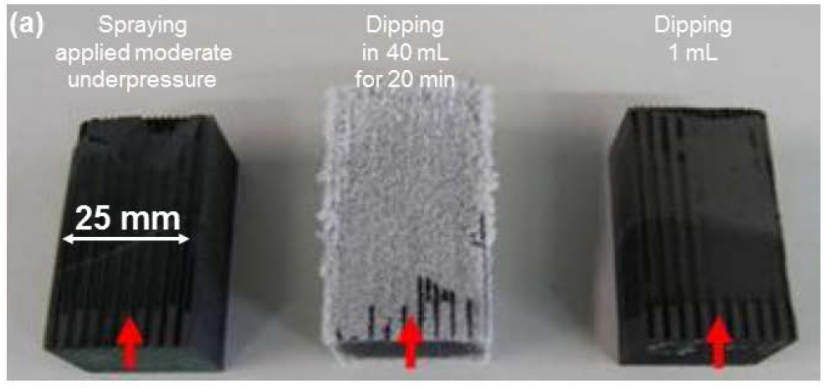

(b)
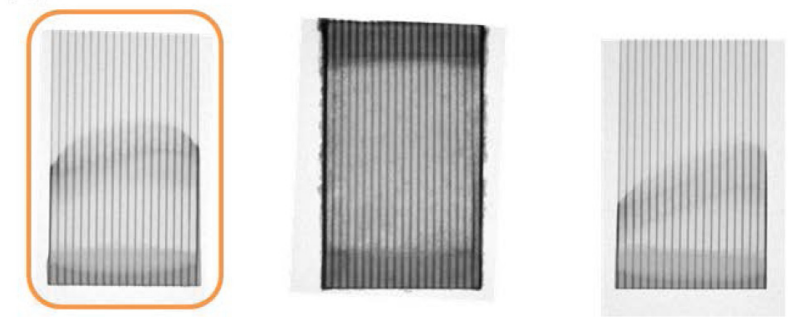

(c)
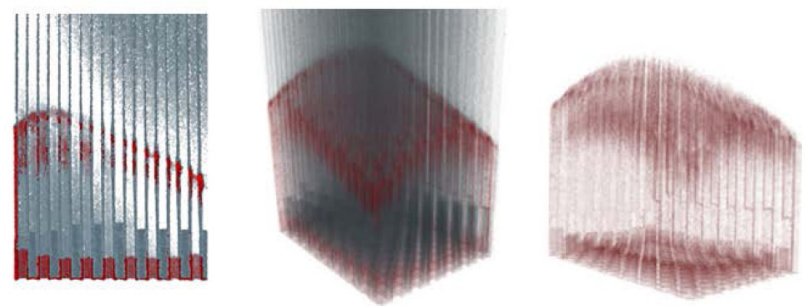

Figure 4. (a) Photograph showing the three used samples and the different ways how the urea solution was applied. (b) Neutron radiography images of the corresponding samples. (c) Neutron tomography results of the urea distribution of the sample indicated with the orange box in (b).

\section{Ash Distribution in a Canned Passenger Car GPF}

In the near future it is foreseen to equip even gasoline engines with particulate filters with the aim to reduce the amount of particulate matter. In Figure 5 we present the neutron tomography results of an identical canned cordierite substrate sample, analyzed in empty state and subsequently after the loading. For the NT measurement 625 projections over an angular range of $360^{\circ}$ were taken with an exposure time of $15 \mathrm{~s}$ per projection. In Figure 5(a) a vertical slice image of the unloaded state and in Figure 5(b) in the loaded state. The magnified detail of the lower part as indicated by the orange box shows clearly the plug ash accumulation. To quantify the amount of ash in each channel, we summed up the ash filling over a length of $6 \mathrm{~mm}$, as indicated in the magnified image in Figure 5(b). The result of the individual ash height in each channel is given in Figure 5(c). The three-dimensional representation of the ash loading is shown in Figure 5(d).

In Figure 6 we analyzed the obtained tomography data from Figure 5 in a different way [ [ $\underline{3}$. This allows us, beside the detection of plug ash, to obtain the information of ash deposits along and onto the filter walls. In order to compare the ash loading along the filter substrate, the mean grey value of each horizontal slice (compare, inlet in Figure 6) along the loading direction were calculated. Higher grey values indicate a stronger loading. It can be directly seen from the evaluated profiles that the amount of ash deposits throughout the filter, increases from the inlet side to the outlet side. This contribution can be referred as the wall ash. At the end of the substrate the pronounced plug ash peak is visible. Moreover, the homogeneity of loading of the ash deposits can be evaluated from the loaded profile as the unloaded profile shows a constant grey value from the inlet to the outlet. Two peaks are visible in the unloaded profile, one at the inlet position and one at the outlet position, respectively. Both represent the inlet and outlet plugs. (a)

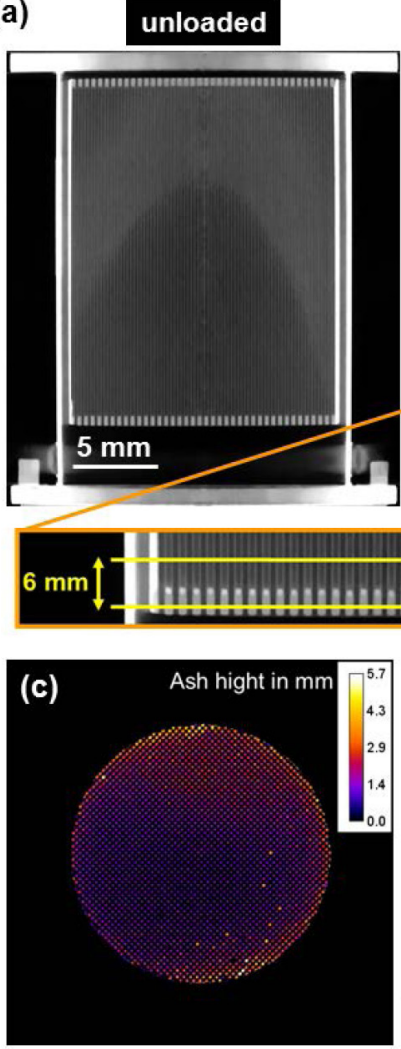

(b)

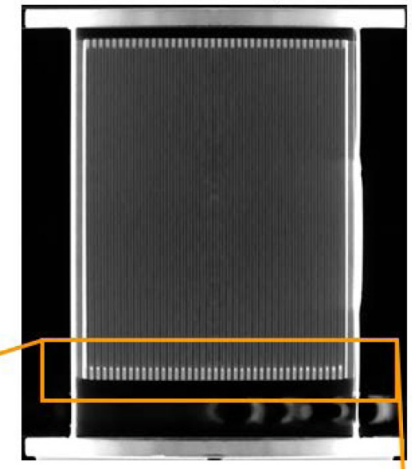

Figure 5. (a) Vertical tomography slices of canned and unloaded GPF. (b) Vertical tomography slices of the same canned GPF with loading. A zoom-in of the ash residuals is shown. (c) Analyzed ash height per filter channel by summing up the bottom $6 \mathrm{~mm}$ as indicated in (b). (d) 3D representation of (c).

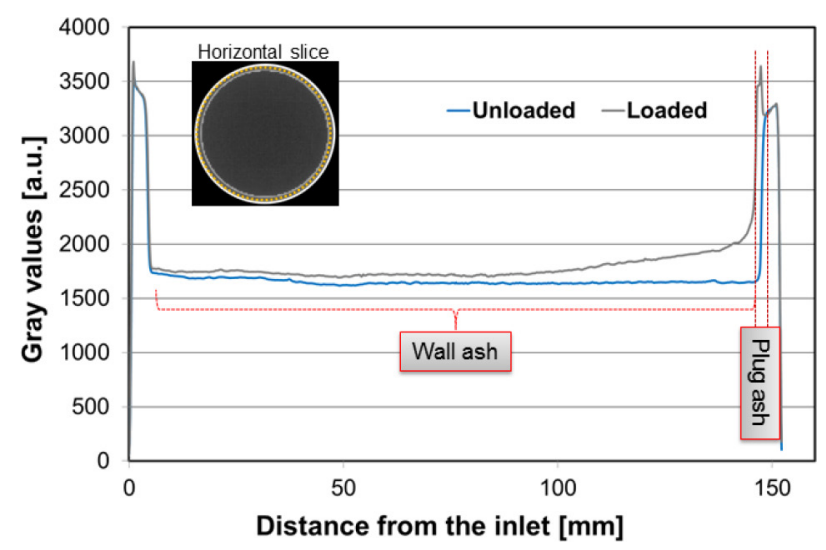

Figure 6. Line profiles along the vertical direction of the unloaded (blue) and loaded (gray) GPF. The higher the grey level, the more abundant are the ash deposits. Each line data point is obtained by averaging the grey values of each corresponding tomography slice within the area as indicated by the dashed orange circle in the inlet of one representing slice. Beside the detected plug ash the similar amount of ash is found on the substrate walls all through the filter substrate. 


\section{Ash Residuals of a Truck DPF after Cleaning with Different Techniques}

Truck and heavy-duty vehicles DPFs are commonly cleaned to remove the residual ash loading. Here we show how the neutron imaging technique was used for a comparison of two different filter cleaning methods to analyze the residual ash deposits. The first method uses an air jet based technique whereas the second one is based on the more recently developed Xpurge technique [5]. Two canned 12 inch cordierite filters were investigated. Both filters were loaded under the same conditions. Filter 1 was primarily cleaned using the air jet technique and Filter 2 with the Xpurge technique. The neutron radiography results are shown in Figure 7(a), (b), respectively. For the NT measurement 1125 projections over an angular range of $360^{\circ}$ were taken with an exposure time of $4 \mathrm{~s}$ per projection. Already in a single radiography image, the Xpurge cleaned filter looks cleaner. In a second step, the air jet cleaned filter was subsequent cleaned by the Xpurge technique, and the result is shown in Figure 7(c), where an improvement of the cleaning is visible. Figure 7(d) shows the second cleaning of filter 2 with the Xpurge technique, where not much changes in contrast to the first cleaning.

\section{Filter 1}

(a) Air jet cleaning

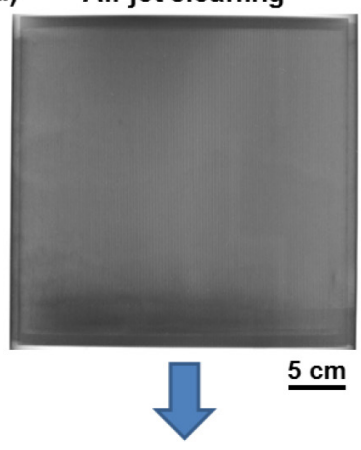

(c) Xpurge cleaning

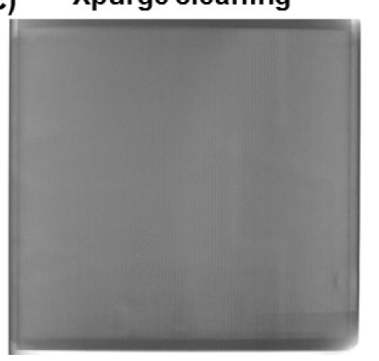

Filter 2

(b) Xpurge cleaning

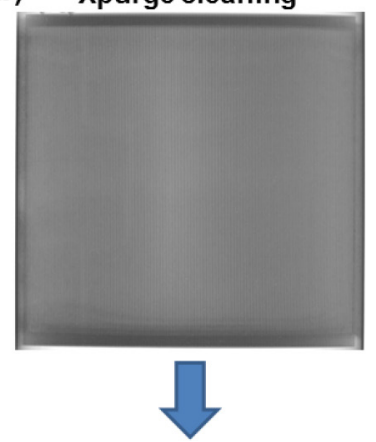

(d)

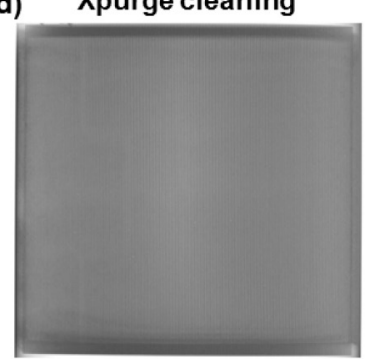

Figure 7. Neutron radiographies of two canned truck DPF. (a) Filter 1 cleaned with an air jet technique. (b) Filter 2 cleaned with the Xpurge technique.(c) Filter 1 additionally cleaned with the Xpurge technique. (d) Filter 2 again cleaned with the Xpurge technique.

For a more quantitative analysis of the residual ash and cleaning efficiency we acquired on both filter after each cleaning procedure a tomography data set. The processing chain to finally obtain the total ash loading height, similar as what has been presented in Figure 5, is shown in Figure 8. Starting with the reconstruction of the recorded $2 \mathrm{D}$ projections yields the $3 \mathrm{D}$ attenuation slices. Applying a structure filter on the $3 \mathrm{D}$ attenuation slices results in the information of individual channel loading slices. From this point one can visualize the ash deposits in each channel by the corresponding volume rendering. For the final quantitative information of the total ash height in the substrate we summed up the data from the channel loading slices which results finally in the total 3D ash loading height in the whole filter.
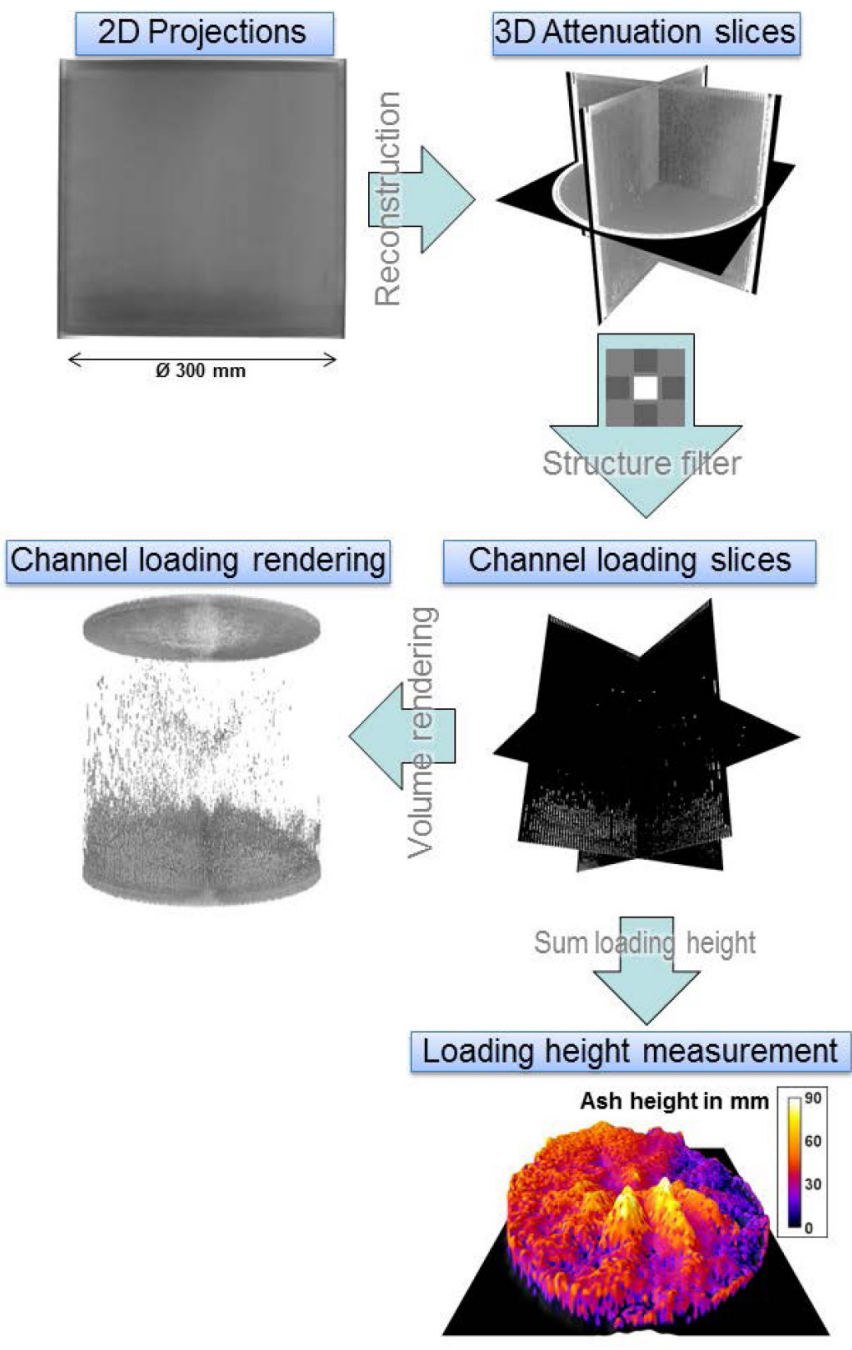

Figure 8. Processing chain to obtain the ash height in a canned truck DPF. The reconstruction of the $2 \mathrm{D}$ projections yield the $3 \mathrm{D}$ attenuation slices. The application of a dedicated structure filter leads to the information of the channel loading slices and by a further volume rendering the channel loading rendering is obtained. Doing the sum loading height will finally results in the quantitative information about the ash height in the loading height measurement.

The results of the total ash heights of Filter 1 and 2 after the different cleaning steps with the different techniques are shown in Figure 9. In the first cleaning step in Figure 9 (a), (d), the Xpurge technique shows clearly less ash residuals as for the air jet cleaning. In the second step both filters have been cleaned using the Xpurge technique. The additionally cleaning of Filter 1 with the Xpurge technique shows a further cleaning improvement as seen in (b), whereas for the second time Xpurge cleaned filter does not show any improvement. The ash height difference for both filters after the 2 step cleaning is shown in (c) and (f). 
(a)
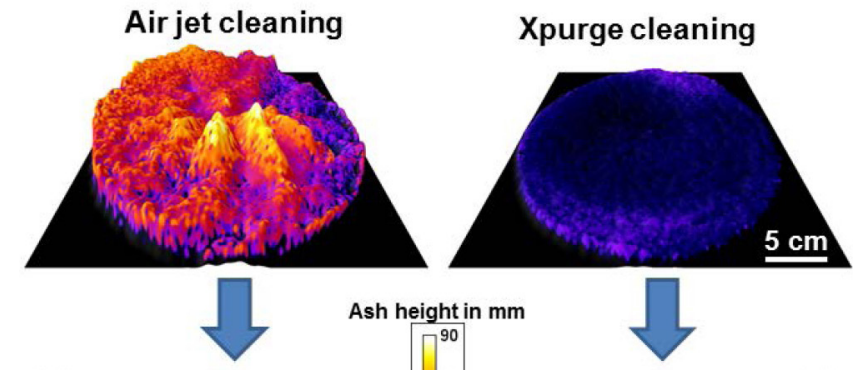

(b)

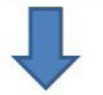

Ash height in $\mathrm{mm}$
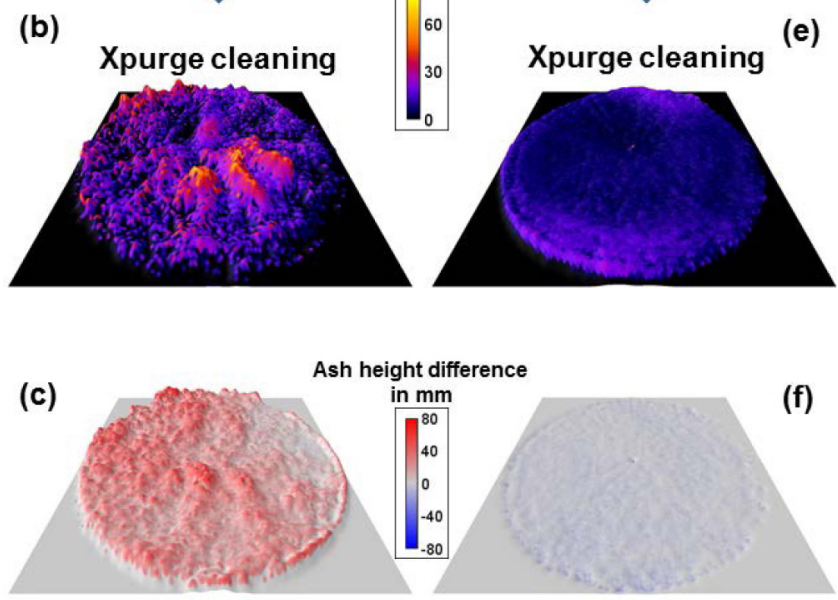

Figure 9. Comparison of two ash cleaning techniques. (a) Ash height of the remaining ash after an air jet cleaning of filter 1 . (b) Follow-up Xpurge cleaning of filter 1. (c) Showing the ash height difference. (d),(e) Filter 2 which is twice cleaned with the Xpurge technique. (f) Showing the ash height difference.

\section{Summary/Conclusions}

In conclusion we have shown how neutron imaging can yield detailed information about ash, soot, urea and coating distributions in canned DPF of passenger cars and trucks and GPF of passenger cars. The advantage of neutrons in contrast to X-rays is that the metallic canning does not constitute an obstacle for 2D and 3D neutron imaging experiments. The sensitivity of neutrons for hydrogen and the more element specific attenuation makes them an ideal probe to study non-destructively canned exhaust after-treatment system components.

\section{References}

1. Zandhuis, J., Finney, C., Toops, T., Partridge, W. et al., "Nondestructive X-ray Inspection of Thermal Damage, Soot and Ash Distributions in Diesel Particulate Filters," SAE Technical Paper 2009-01-0289, 2009, doi:10.4271/2009-01-0289. )

2. Grünzweig, C. e. al., " Progress in industrial applications using modern neutron imaging techniques," Physics Proceedings 43, 231-242 (2012)..

3. Grünzweig, C., Mannes, D., Kaestner, A., Vogt, M., "Visualizing the soot- and ash distribution in diesel particulate filters using neutron imaging" MTZ 73(4):326 (2012).

4. https://www.youtube.com/watch? $\mathrm{v}=\mathrm{A} 4 \mathrm{kWCP} 39 \mathrm{t} 6 \mathrm{U} \&$ feature $=\mathrm{pl}$ ayer embedded.

5. http://www.ceramex.com/xpurge-dpf-cleaning/

\section{Contact Information}

Dr. Christian Grünzweig Scientist, Neutron Imaging and Activation Group Laboratory for Neutron Scattering and Imaging (LNS)

Paul Scherrer Institut

CH-5232 Villigen PSI

Switzerland

phone: +41563104662

christian.gruenzweig@psi.ch

\section{Definitions/Abbreviations}
NI - Neutron imaging
DPF - Diesel particulate filter
GPF - Gasoline particulate filter
NO - Oxide of nitrogen
PSI - Paul Scherrer Institut
SCR - Selective catalytic reduction
DEF - Diesel exhaust fluid
SiC - Silicon carbide

The NI beam lines at PSI are available for industrial users after consultation to perform the here described or similar experiments.

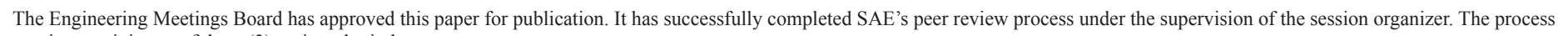
requires a minimum of three (3) reviews by industry experts.

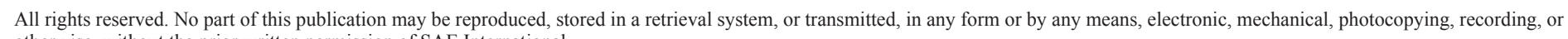
otherwise, without the prior written permission of SAE International.

Positions and opinions advanced in this paper are those of the author(s) and not necessarily those of SAE International. The author is solely responsible for the content of the paper.

ISSN 0148-7191

http://papers.sae.org/2016-01-0985 